\title{
Improving the use of phlebotomy services in a paediatric inpatient setting
}

\author{
Jacqueline Pitchforth, Claire Lemer
}

Guys \& St Thomas' Trust, London, UK

\begin{abstract}
The aim of this project was to improve the use of the current Paediatric Phlebotomy service. The main problem identified was the lack of a system to notify medical teams of unsuccessful blood requests. A phlebotomy process map was constructed to help analyse reasons why failure to notify occurred. Pre and post intervention audits were conducted using the number of unsuccessful blood requests notified as a baseline measurement. The initial intervention was modified based on feedback during the process. A $44 \%$ improvement in the number of unsuccessful blood requests notified and a potential cost savings of $£ 6240$ was demonstrated in the post-intervention audit. Further work is required as to whether these improvements can be increased, sustained and be cost effective.
\end{abstract}

\section{Problem}

The Evelina Children's Hospital offers a twice daily phlebotomy service on the General Paediatric ward. At present this service is not as useful as it could be. Currently blood tests are ordered on the electronic patient record (EPR) for a specified date and the phlebotomy service prints the labels, takes a blood sample from the child and sends the blood to the laboratory. During this time the EPR order reads as 'pending collection' until the blood sample actually reaches the laboratory. Usually the blood samples are sent as a batch together at the end of a phlebotomy list.

If the phlebotomists are unsuccessful in obtaining a blood sample from the child for various reasons, such as, the child wasn't present, the anaesthetic cream was not applied or the venepuncture itself was unsuccessful, then there is no system of letting the medical team know this has not been done. Typically junior doctors discover at the end of day that the blood was not taken and usually have to take it themselves. This can lead to inefficient use of junior doctors' time, delay in decision-making and delay in the time of discharge. This potential delay in discharge can generate:

1. Increased workload for after-hours services such as pharmacists, discharge summaries written by on-call teams who don't know the patients

2. Late night discharges

3. Increased length of hospital stay as patient discharges are sometimes delayed until morning

4. Reduced patient satisfaction

5. Frustration of junior doctors

6. Poor implementation of service provision.

\section{Background}

A lesson learnt from other successful quality improvement projects (QIP) such as 100000 lives saved (1) was the need to 'get to the field'. In other words, gain an understanding of the processes and people involved in the area targeted for improvement. So, with consent, a phlebotomist was shadowed for a morning and a process map constructed on 1 June 2013.

This process map showed that a work-list on EPR was printed off at 0800 and 1400 by the phlebotomists for their AM \& PM lists respectively for the three wards that they serviced. The existence of this work-list was new information to myself and most of my colleagues. The Renal ward was always done first as the Tacrolimus levels were considered the most time critical blood test. The phlebotomists then walk upstairs to the paediatric specialities ward and finally the General Paediatrics ward. They were only supposed to spend 10-15 min per child and if there were any problems to refer back to the doctors and move on. In practise, if a test couldn't be done because the child was at school, away for further investigations or didn't have cream applied, then the phlebotomists placed them on the PM list thus inadvertently delaying the results of the blood tests.

If a test was not done, then the phlebotomists notified the nurse looking after the child but did not notify the medical team. The phlebotomists were unaware if child was surgical, general paediatric, ENT, orthopaedic, neurology, cardiac or renal, and felt that to contact up to seven different SHOs would create too much work. After discussion with the phlebotomists, it was felt that bleeping the nurse in charge on the General Paediatric ward (as the most time sensitive ward) was an achievable goal. After the bloods were taken, they were transported by porters rather than the pneumatic Pod system. Unless the nurse looking after the child informed the medical team, there was no way of knowing whether the blood request had been successful until the sample arrived in the laboratory.

\section{Baseline Measurement}

Measurement allows us to determine the current position, providing the means by which we can evaluate how successful we have been in our efforts (2). In order to determine whether any intervention was successful, it needed to be measurable, and defined over a certain time period. The baseline measurement used in this project was the number of unsuccessful blood requests notified to the medical team. It was measured in both pre and post intervention 
audits to determine if the intervention was successful.

A one week, twice daily, pre-intervention audit was undertaken from 8-12 July 2013 using the number of unsuccessful blood tests notified in both morning and afternoon phlebotomy rounds as a baseline measurement. The proposal was presented at the morning ward round to the attending clinician and junior medical staff, though no direct involvement was required at this stage. The preintervention audit showed that 30 blood tests were requested of which 11 were unsuccessful and only three of these were notified. So the pre-intervention notification of unsuccessful blood requests was $27 \%$.

\section{Design}

Construction of a Phlebotomy process map allowed analysis of the cause of failure to notify the medical team and also highlighted a number of problems with our initial intervention. For example, there was a need to change from informing the medical team to informing the nurse in charge as a single point of contact, in order to reduce the burden on the phlebotomist, and improve compliance. It also suggested that more people needed to be involved and also emphasised the need for information sharing on the existence of the EPR work-list.

The initial design involved establishing a baseline notification rate of unsuccessful blood requests through a pre-intervention audit, then performing an intervention and re-auditing the process to see whether the intervention was successful in producing an improvement in notification rates. The original intervention idea had been to ask the phlebotomist to inform the medical team directly. However after the process mapping it was evident that this was unlikely to be successful and the intervention was modified to incorporate the phlebotomists' feedback.

As the new intervention required the collaboration of the nurse in charge as an intermediate person and also medical staff with whom the Nurse in charge would liaise with, the most appropriate forum to seek consent was through the MDT (Multidisciplinary Team) grand round. Feedback from colleagues after the proposed interventions were presented at the MDT and led to further suggestions for improving the use of the phlebotomy services. These included:

1. A print-out for the nurses at handover so they would know which children were due to have blood tests and so that cream was applied at 0800 for AM bloods and 1400 for PM bloods

2. The medical team to be aware of list on EPR and to know that the phlebotomists place a copy with times of bloods taken or reasons why not in the treatment room.

Further suggestions included updating the phlebotomy service managers with the proposed interventions, the inclusion of existence of the EPR work-list in the induction programmes of incoming junior doctors to order to sustain improvements and for the nurse in charge to disseminate the information to other nurses.

Thus the scope of the intervention broadened and included information sharing and communication with a diverse range of people. The actual intervention still involved the phlebotomists bleeping the nurse in charge in order to notify the number of unsuccessful blood requests after AM and PM phlebotomy rounds and the nurse in charge to inform the medical team at end of ward round handover of the unsuccessful blood requests. The paediatric medical teams then needed to decide whether to do bloods themselves or place on the PM list. A polite reminder notice with the nurse in charge contact details was also placed on the phlebotomy work trolley.

\section{Strategy}

The Plan, Do,Study, Act (PDSA) cycle is an important tool in the Quality Improvement process (3). There were three PDSA cycles during this project, the first after reviewing the Phlebotomy Process map, the second after the MDT and the third after the postintervention audit analysis.

The first PDSA cycle involved changing who the phlebotomist notified to minimise additional workload, as well as broadening scope of intervention to include, educating doctors on the existence of a EPR work-list, and how to access it. The original intervention design involved the phlebotomists directly informing the medical team of any unsuccessful blood requests, and then auditing the results. After completing a phlebotomy process map, it was evident that asking a busy phlebotomist to contact up to seven medical teams was unreasonable in view of their heavy workload and therefore other people would need to be involved. Knowledge of the existence of an EPR work-list could potentially improve the notification rates as timely application of anaesthetic cream could be achieved if the nurses knew which children required blood tests. It was felt the best way to disseminate information to a wide audience and gain their cooperation and feedback was to present the proposed audits and intervention at the MDT grand round. The phlebotomy service managers were also kept informed about the proposed interventions.

The second PDSA cycle involved modifying the intervention to incorporate suggestions which arose from the MDT. The proposed intervention was met with great support, and knowledge of the EPR work-list welcomed. The nurse in charge was happy to participate and requested a copy of the proposal so she could keep the ward sisters updated. In addition, she would ask the nurses to look up the EPR worklist each day so that cream could be applied on time to the relevant children in order to improve successful blood request rates. The consultant in charge of junior doctors induction was keen for the information regarding the EPR work-list to be included in future induction programmes. The feedback was used to modify the intervention as well as providing areas for future examination such as sustained improvement.

The third PDSA cycle arose after discussion with phlebotomists and analysis of post intervention audit data. The post intervention audit demonstrated an improvement in the notification of unsuccessful blood requests, but highlighted a misunderstanding regarding blood requests from long lines which if corrected could potentially improve notification rates further. It was also unknown whether these 
improvements would be sustained and whether they would results in real cost savings. Further work is required to determine whether improvements are possible if the misunderstandings are clarified. Further analysis of potential cost savings with regard to reducing hospital stays and earlier discharges also needs to be quantified.

\section{Post-Measurement}

Data for the post intervention audit was collected twice daily for one week from 29 July-2nd August 2013. There were a total of 46 blood requests of which 21 were unsuccessful and 15 of these were notified to the nurse in charge giving a notification rate of $71 \%$. This was an improvement of $44 \%$ from the $27 \%$ measured in the preintervention audit.

In the pre-intervention audit, the most common reason for unsuccessful blood request was not having anaesthetic cream applied 36\% (3/11). In comparison, in the post intervention audit, only $9.5 \%(2 / 21)$ of the blood requests were unsuccessful due to anaesthetic cream not being applied in time. The reasons for failure to notify in the pre-intervention audit were varied. For the three unsuccessful requests that were notified - one was "cancelled by the doctors", the second was told that it was "not required" by the doctors and the third was "doctors to do". However, in the post intervention audit, 5 of the 6 unsuccessful blood requests that weren't notified were related to central lines and only one to cream not being applied. After further investigation, it was discovered that since phlebotomists do not take blood from central lines, they assumed the nurses would do it and therefore did not notify the nurse in charge of such unsuccessful requests.

Based on our results, if one unsuccessful blood test reported earlier resulted in one less day as a hospital inpatient and saved 1 hour of a junior doctor's time chasing blood results then a potential cost saving of $£ 530$ could be made. Conversely, if every notified unsuccessful blood request resulted in a reduced hospital stay of one day per patient and one day of doctors time was saved then savings of up to $£ 6240$ ( 12 nights and 1 day FY2 time) could be made. However these savings are speculative and further work would be required to ascertain the true savings from improved notification rates.

See supplementary file: ds2175.xls - "Pre \& post intervention audit data"

\section{Lessons and Limitations}

One exciting lesson learnt from this project is that real change is possible but it is a collaborative effort with communication and flexibility at the heart. For example interventions have to be practical. Phlebotomists are under significant time pressures and asking them to determine which team a patient belongs to and potentially trying to bleep up to 7 different doctors is too time consuming. Making one bleep to the nurse in charge is achievable.

A recent paper quoted that "communication breakdowns are involved in the great majority of adverse patient outcomes" (4).
Effective communication is probably one of the key ingredients to improving patient outcomes. Many assumptions, particularly related to lack of knowledge of how others work, had been made in the process of requesting a blood test on EPR. Firstly the phlebotomists assumed that the presence of EPR work-list was common knowledge when in fact it was user specific information and very useful when it was disseminated. The construction of process maps was useful for understanding what people actual did as well as eliciting assumptions made about how others work which was extremely helpful in planning the intervention. Secondly, doctors assumed that if blood tests were ordered early in the morning that the results would be available early afternoon. This was not the case especially if the phlebotomists were busy on other wards first or returned in the afternoon because cream was not applied in time. A third assumption arose from blood requests ordered from long lines. Presumably the doctors making the requests either didn't know a child had a long line or expected the phlebotomists to do them. However, since the phlebotomists do not sample from central lines they assumed that the nurses would take the blood and therefore didn't notify anyone when they were not done.

Another lesson learnt in this project was that quality improvement is a process of change with one improvement opening the door to the next round of improvements. This project is already paving the way for future projects to see whether further improvements are possible by clarifying the misunderstanding over long lines, whether the improvements seen are sustained and to quantify the cost savings associated with the improvements.

The main limitation to this project is the small number of samples involved in the audit. It is possible that the results may be a product of chance. The general application of these results may be limited to busy general paediatric wards as other paediatric specialties such as neurology were less concerned about the timeliness of their results and acute adult wards don't routinely use anaesthetic cream. However some of the principles such as an EPR work-list and long line issues may be relevant to other areas.

\section{Conclusion}

The aim of this project was to improve the use of the phlebotomy services in a paediatric inpatient setting. The Post intervention audit demonstrated that the intervention was successful in producing a $44 \%$ improvement in the number of unsuccessful blood requests notified. The phlebotomy process map played an important role in modifying the original intervention design and also highlighting the need for wider involvement of medical and nursing staff. Further improvements in notification rates may be gained from clarifying the confusion over long line sampling and timely application of anaesthetic cream by nursing staff. Ongoing education at the induction of new doctors maybe a method for disseminating institutional knowledge thereby sustaining improvements and avoiding a deterioration in notification rates with time.

Several questions were raised by this project including whether the improvements in notification rates actually lead to earlier discharges for those previously held up by delayed blood tests as well as the true cost savings and whether sustained and increased 


\section{BMJ Quality Improvement Reports}

improvement is possible. In conclusion, this project demonstrated a successful intervention but raised issues that highlight the need for ongoing audit and further QI projects.

\section{References}

1. Berwick D, Calkins DR, McCannon CJ, Hackbarth AD. The 100000 Lives Campaign. Setting a Goal and a Deadline for Improving Health Care Quality. JAMA.

2006;295(3):324-327. doi:10.1001/jama.295.3.324.

2. Faculty for Healthcare Improvement Report . Making a real difference to healthcare in Wales. The Journey so far. 2009. Available from http://www.1000livesplus.wales.nhs.uk/opendoc/179357

3. Taylor MJ, McNicholas C, Nicolay C, Darzi A, Bell D, Reed JE. Systematic review of the application of the plan-do-study-act method to improve quality in healthcare. BMJ Qual Saf doi:10.1136/bmjqs-2013-001862

4. Lee P, Allen K, Daly M. A 'Communication and Patient Safety' training programme for all healthcare staff: can it make a difference? BMJ Qual Saf 2012;21:84-88 doi:10.1136/bmjqs-2011-000297

5. Davidoff F, Batablen P, Stevens D, Ogine G, Mooney S. Publications guidelines for quality improvement in health care: evolution of SQUIRE project. Qual Saf Health Care. 2008; 17 Supp 1.

\section{Declaration of interests}

Nothing to declare 